Semin Liver Dis. 1985 November ; 5(4): 394-401. doi:10.1055/s-2008-1040638.

\title{
Retransplantation of the Liver
}

\author{
BYERS W. SHAW Jr., M.D., ROBERT D. GORDON, M.D., SHUNZABURO IWATSUKI, M.D., \\ and THOMAS E. STARZL, M.D., Ph.D. \\ Department of Surgery, University of Pittsburgh Health Center, University of Pittsburgh, \\ Pittsburgh, Pennsylvania
}

\begin{abstract}
Improvements in operative technique, immunosuppression, and organ procurement have done much to enhance the probability that the liver recipient will survive long term. Nevertheless, failure of an hepatic allograft continues to be a serious risk facing the liver recipient. Because no effective method of extracorporeal support is available for these patients, undergoing retransplantation is the only alternative that offers the potential for long-term survival.

In the Pittsburgh experience, the need for retransplantation has arisen in about 20 to $25 \%$ of liver recipients. It can occur as the result of three basic causes - primary nonfunction, rejection, or as a result of technical difficulties — and can present in one of several different settings. The present report is a review of a previous study that examined the subject of retransplantation in depth. ${ }^{1}$
\end{abstract}

\section{METHODOLOGY AND DATA BASE}

A total of 70 patients of the combined Denver and Pittsburgh series required retransplantation. Of these, 21 were from the group of 170 patients transplanted in Denver between March 1, 1963, and February 3, 1980, under azathioprine and prednisone immunosuppression. This group was not examined in detail, but their survival data were compared with the group of 49 retransplanted patients operated on in Pittsburgh through August 1, 1984.

In a retrospective review of patient records, the major causes of hepatic graft failure virtually always could be assigned to one of the three main categories just enumerated. A diagnosis of primary nonfunction was made if a graft never showed evidence of initial function following its transplantation. The signs might include those of profound hepatic failure (severe hypoglycemia, deep coma, renal failure, marked coagulopathy, acidosis, and shock), or other less obvious signs of irreversible hepatic damage (massive increases in serum transaminase levels, unrelenting daily increases in serum bilirubin levels, mental confusion, persistent coagulopathy, or persistent renal insufficiency without other evident causes).

If a graft failed secondarily, following initial evidence of acceptable function, the cause was assigned to one of two other categories. Rejection was diagnosed if a chart review offered clinical support for that diagnosis or if histopathologic review of the removed graft showed evidence of rejection. Technical failures include, primarily, vascular thromboses and, less frequently, complications of biliary reconstruction or other errors in surgical technique. 


\section{RESULTS}

\section{Relative Incidence of Retransplantation}

Prior to the introduction of cyclosporine, retransplantation was employed infrequently. Only 21 of 170 patients (12.4\%) received second grafts. In the cyclosporine-treated group, of 323 total operations, $69(21.4 \%)$ were retransplants. Of the 69 retransplants, 60 were for second and nine for third grafts. Yearly breakdown of the relative frequency of retransplantation is shown in Figure 1.

Retransplantation did not occur with any difference in frequency that was dependent on the original cause of liver disease (Fig. 2). In addition, the causes of retransplantation did not occur with any difference in frequency among the various primary disease categories (Fig. $3)$.

\section{Relative Frequencies of the Causes of Retransplantation}

As indicated in Figure 4, rejection was the most common reason for retransplantation, occurring in 28 of the 49 (57\%) patients ( 15 of 24 adults and 13 of 25 children). Technical failures accounted for graft failure in $10(20 \%)$ patients overall: 2 adults $(8 \%)$ and 8 children $(32 \%)$. The greater frequency of technical failures in children is statistically significant $(\mathrm{p}<$ $0.05)$.

Primary nonfunction occurred in 11 of the 49 patients (22\%). It was the second most common reason for retransplantation in adults ( 7 of 24 , or $29 \%$ ) and the least common in children (4 of 25 , or $16 \%)$.

\section{Survival Data}

Life-table methods were used to produce and compare survival curves for various groups of patients in the study. Figure 5 shows the difference in survival following retransplantation in the azathioprine- and cyclosporine-treated groups. The former group had a 14\% 1-year survival and no patient survived beyond 16 months. The cyclosporine group obtained a $49 \%$ survival rate for 6 months through 2 years (no patient has died more than 6 months after retransplantation).

Figure 6 shows a breakdown of survival curves comparing primary with secondary grafting in children and adults. All patients who avoided retransplantation fared better than retransplanted patients, with $68.5 \%$ of the former and $49 \%$ of the latter surviving at least 1 year $(\mathrm{p}=0.014)$. The 1-year survival rate in adults requiring retransplantation was $42.3 \%$ compared with $63.5 \%$ in those with only primary grafting $(\mathrm{p}=0.026)$. The parallel rates in children were $56.5 \%$ for those retransplanted and $76.8 \%$ for those receiving only one graft $(\mathrm{p}=0.042)$. The survival figures were better for children than adults in the primary group ( $\mathrm{p}$ $=0.038)$ but not in the retransplanted group $(\mathrm{p}=0.159)$.

No differences in survival following retransplantation were evident in an examination of survival curves for different primary disease categories. Children with biliary atresia fared the best and adults with sclerosing cholangitis the worst, but the difference between these two extremes was not significant $(\mathrm{p}=0.074)$.

Figure 7 shows the survival curves for the different causes of retransplantation. Both the rejection and the technical failure groups fared better than the primary nonfunction group $(\mathrm{p}$ $<0.03$ for both comparisons). 


\section{Overall Impact of Retransplantation}

The overall effect of retransplantation on survival data is shown in Figure 8. The lower curve assumes that all patients not receiving retransplantation would have died within a few weeks of their retransplantation date. A further illustration of the effect of retransplantation is shown in Table 1. Because of an aggressive attitude toward early retransplantation in Pittsburgh, only seven patients could be identified during the study period who should have been retransplanted but were not because an appropriate donor was never available. In five of these patients, the need for retransplantation arose as an emergency situation, requiring a new organ within hours to a few days. All five of these patients died as a direct result of hepatic failure. The two patients, with chronic rejection, illustrate the other danger of waiting too long for retransplantation. Both were children who died of sepsis resulting from overimmunosuppression in the presence of increasing liver failure while waiting for an appropriate donor.

\section{Operative Blood Losses}

The mean operative blood losses for retransplantation procedures compared with primary grafting procedures are shown in Table 2. Considerably less blood was lost during the average retransplant operation than during the first operation. This is true in both adults and children, although in order to have a statistically significant difference in children, one must eliminate from consideration three cases of arterial thrombosis.

\section{Influence of a Positive Cytotoxic Cross-Match}

Information about the presence of donor-specific, cytotoxic T-cell antibodies in the serum of recipients was available in 148 patients. Cross-matches were not done in another 92 cases. Table 3 shows the relationship between a positive or negative cross-match and the requirement for retransplantation. A positive cross-match was not associated with a higher incidence of retransplantation. In addition, the incidence of a positive cross-match was not different between those patients with primary grafting compared with those undergoing retransplantation. As noted in a previous report, the incidence of a positive cross-match increased from about 25 to $50 \%$ at the time of the first retransplantation and to $75 \%$ at the time of tertiary grafting. ${ }^{2}$

\section{Tertiary Transplantation}

At the time of the study, a total of nine patients had been given three sequential hepatic allografts (Table 4). Five of these patients are still alive and continue to do well. Three are male children and two are adults.

The four who died were all patients for whom third transplants were attempted in rather desperate situations. As pointed out in an accompanying article in this issue of Seminars, survival following retransplantation has not been possible when the patient is taken back to the operating room in stage III or IV coma as the result of failure of the previous graft.

\section{LESSONS FROM THE CURRENT EXPERIENCE}

\section{Technical Considerations}

Several concepts should be kept in mind when planning for a retransplantation.

The usefulness of vascular grafts procured at the time of the donor hepatectomy has been noted previously. ${ }^{3}$ This proves to be even more important in cases of retransplantation resulting from a vascular thrombosis, since an alternate method of arterial or portal venous reconstruction may be required. ${ }^{4,5}$ 
In preparing the upper vena cava for anastomosis to the new hepatic graft, the surgeon may obtain extra length by leaving the original anastomosis intact and sewing to a short cuff of vena cava from the first liver (Fig. 9). Even if the sutures encompass the first anastomosis, the extra length provided by the cuff of vein may prove to be critical.

The success of biliary reconstruction during a retransplantation depends on careful assessment of the suitability of the recipient tissue to which the new bile duct is to be sewn. If a duct-to-duct anastomosis is planned, this means making certain that all tissue from the previous donor duct be removed and that the remaining recipient bile duct is viable and still long enough to allow connection to the new donor duct without tension. If any doubts arise, choledochodochostomy should be abandoned in favor of anastomosis to a Roux limb of jejunum. In reusing a jejunal limb, the first site of duct anastomosis should either be excised or closed primarily and a new site prepared elsewhere along the limb for the duct anastomosis.

In general, the difficulty of the recipient operation may vary greatly, depending on the time interval since the previous transplant, the cause of retransplantation, and on whatever might be responsible for the density and extent of adhesion formation. As a rule, care should be taken to avoid overly aggressive blunt dissection, since the walls of blood vessels or suture lines often prove to be the weakest points in the areas being stressed. The best alternative is to use sharp dissection wherever possible, since any errors that are made will likely be less dramatic and easier to repair than disrupted anastomoses or large rents in vessel walls.

\section{The Timing and Setting of Retransplantation}

The need for retransplantation can occur in one of three settings: emergent, acute, and elective. Most often, success in the emergent or acute setting requires an effective organ procurement system and optimal use of all available donors. Proper planning of elective retransplantation demands balancing the risks of further attempts at saving the failing graft against the risks of reoperation.

Aggressive acute rejection, arterial thrombosis, or primary nonfunction can all lead to such profound hepatic failure that retransplantation is required emergently. Although only three patients of the 49 in this group fell into this category, during the same time period, five others in similar straits died before new donor organs could be located. In the setting of rejection, the emergent need for a new graft was indicated by the development of severe adult respiratory distress and a metabolic acidosis, accompanied by sudden increases in serum transaminase levels. For the two patients in this condition who were successfully retransplanted, the secondary transplants were undertaken within 8 to 10 hours of the onset of signs and symptoms. In the two patients who died when no donors were found, death occurred, despite all attempts to support these patients, within 8 and 18 hours of the onset of symptoms.

When arterial thrombosis was the cause of emergent retransplantation, patients usually presented with signs of hepatic gangrene, including the appearance of air bubbles in the right upper quadrant on plain radiographs of the abdomen (see accompanying article on the dearterialized graft). In all of the patients with this entity in this series, the time from development of signs to retransplantation was somewhat longer than for those undergoing emergency retransplantation for rejection. The two patients who died before new organs could be found also had survived with their infarcted grafts for several days. Hence, arterial thrombosis may afford a little more time than rejection for locating an appropriate donor.

The need for retransplantation in the acute and elective settings occurs primarily as the result of rejection. The decision to go ahead with retransplantation in the acute setting is usually 
made when liver function tests continue to deteriorate persistently over a period of several days, despite adequate immunosuppression or with antirejection therapy. The decision in the elective setting can be somewhat more difficult.

In attempting to make the proper decision about retransplantation, one should bear in mind several conclusions from the previous study. The 1-and 2-year survival rates after retransplantation approach those for primary grafting. All of the deaths have occurred within 6 months of retransplantation, and the major cause of death has been related to sepsis. No patient in a coma from acute failure of an hepatic allograft (all in the emergent setting) has survived long term. On the other hand, the retransplant operation is usually accomplished with less difficulty than the first transplant, with the possible exception of those cases of arterial thrombosis wherein extensive arterial collaterals have had time to form. Finally, failure of a second graft, particularly in the elective setting, does not preclude survival after a third, since five of nine such patients have obtained excellent long-term results.

Before deciding to recommend elective retransplantation, the surgeon must make a careful search for reversible causes of liver dysfunction. Drug toxicity, biliary obstruction, viral hepatitis, and, in particular, intra-abdominal abscess are a few of the more common entities that must be excluded. Liver biopsy may be used to help make the diagnosis of other causes of hepatic failure. One must avoid placing too much emphasis on the interpretation of histologic studies, however, without other evidence to support that interpretation.

Findings that support a decision to retransplant a patient include: (1) signs of a chronic rejection that is not likely to be reversible, such as massive elevations of gamma glutamyl transpeptidase levels or a biopsy showing disappearance of bile ductules, arteriolar thickening, and extensive periportal fibrosis; (2) the persistent elevation of serum bilirubin levels in the first month above $10 \mathrm{mg} / \mathrm{dl}$, which is unresponsive to two full courses of antirejection therapy, particularly if the bilirubin continues to increase each time immunosuppression is reduced to maintenance levels; (3) poor liver function that deteriorates even further whenever immunosuppression is reduced to maintenance levels.

The primary goal in the elective situation should be to avoid maintaining barely adequate hepatic function through the use of overaggressive immunosuppression. The temptation often exists to give frequent intravenous boluses of steroids or to try a series of alternative forms of therapy to avoid retransplantation. If the patient's overall health remains good and neither liver function tests nor biopsies show signs of chronic rejection, such efforts might be rewarded. However, once the clinical condition deteriorates as the result of over-zealous attempts to reverse rejection, the chances of surviving retransplantation diminish markedly.

\section{SUMMARY}

Since the introduction of cyclosporine-prednisone for primary immunosuppression, retransplantation has become a feasible option for patients whose primary grafts are failing, which may result from primary graft nonfunction, intractable rejection, or consequent to technical complications. Although survival of patients requiring second grafts is less good than in those whose initial graft functions well, 2-year survival rates of $49 \%$ have been achieved in retransplanted patients, a record that mandates serious consideration of this approach when the primary graft begins to fail. In general, the retransplant procedure is technically easier, with less blood loss, than is the initial operation. When the reoperation is done electively, it should be done before serious clinical deterioration compromises the chances for success. 


\section{Acknowledgments}

Supported by Research Grants from the Veterans Administration and Project Grant No. AM-29961 from the National Institutes of Health, Bethesda, Maryland.

\section{References}

1. Shaw BW Jr, Gordon RD, Iwatsuki S, et al. Hepatic retransplantation. Transplant Proc. 1985; 17:264. [PubMed: 20354567]

2. Iwatsuki S, Rabin BS, Shaw BW Jr, et al. Liver transplantation against T cell-positive warm cross matches. Transplant Proc. 1984; 16:1427-1429. [PubMed: 6390842]

3. Starzl TE, Halgrimson CC, Koep LJ, et al. Vascular homo-grafts from cadaveric organ donors. Surg Gynecol Obstet. 1979; 149:737. [PubMed: 505253]

4. Shaw BW Jr, Iwatsuki S, Starzl TE. Alternative methods of hepatic graft arterialization. Surg Gynecol Obstet. 1984; 159:490-493. [PubMed: 6387980]

5. Shaw BW Jr, Iwatsuki S, Bron K, et al. Portal vein grafts in hepatic transplantation. Surg Gynecol Obstet. 1985; 161:66-68. [PubMed: 3892734]

6. Starzl, TE. Experience in Hepatic Transplantation. Philadelphia, W.B: Saunders; 1969. (with the assistance of CW Putnam) 
LIVER TRANSPLANTS PER CALENDAR YEAR

$1980-1984$

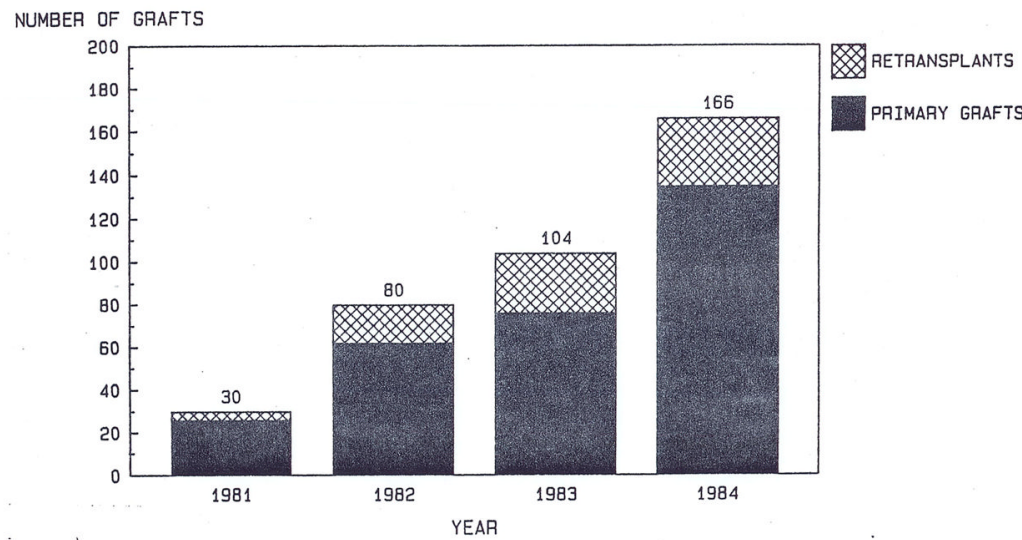

FIG. 1. Liver transplants per calendar year

(Reproduced with permission from Shaw et al. ${ }^{1}$ ) 


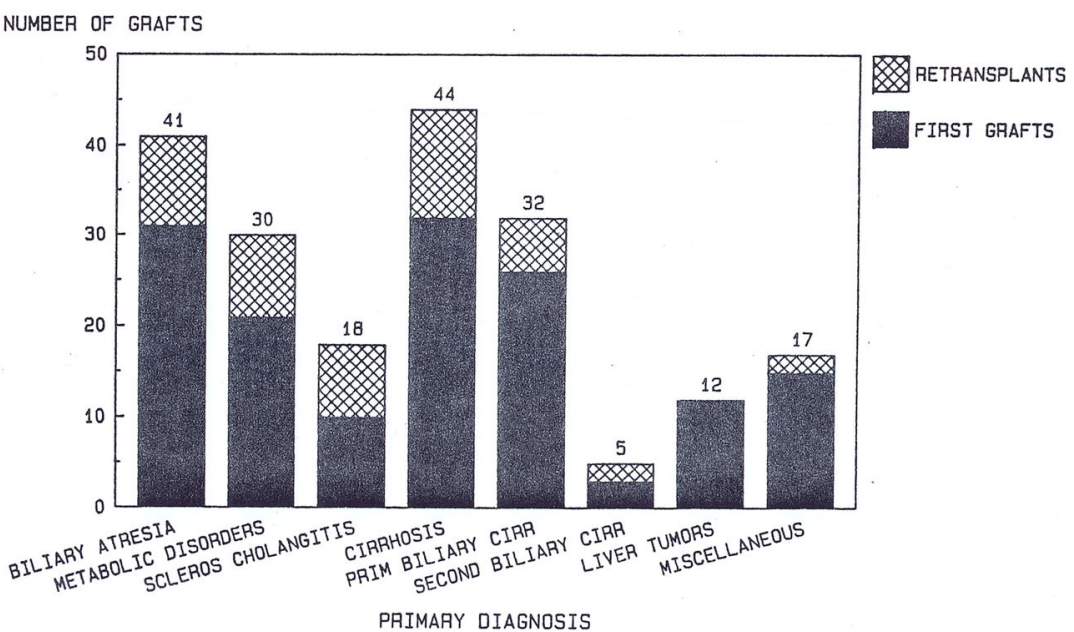

FIG. 2. Liver retransplantation with cyclosporine and prednisone: primary disease categories (Reproduced with permission from Shaw et al. ${ }^{1}$ ) 


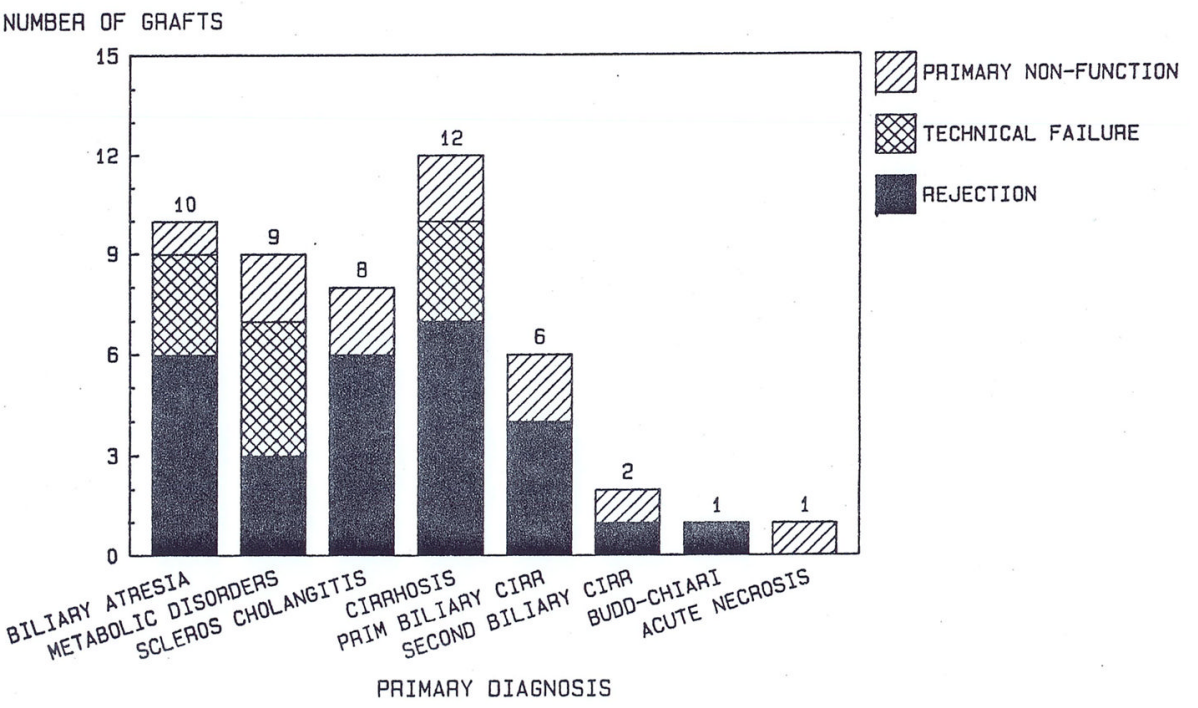

FIG. 3. Indications for liver retransplantation: cyclosporine era

Primary nonfunction in patients, technical failure in 10, and rejection in 28. (Reproduced with permission from Shaw et al. ${ }^{1}$ ) 


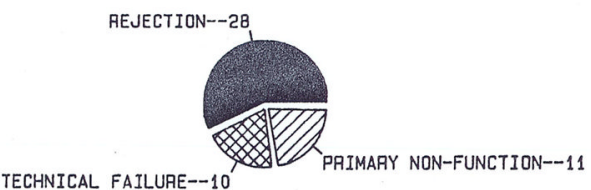

$$
\begin{gathered}
\text { ALL PATIENTS } \\
N=49
\end{gathered}
$$
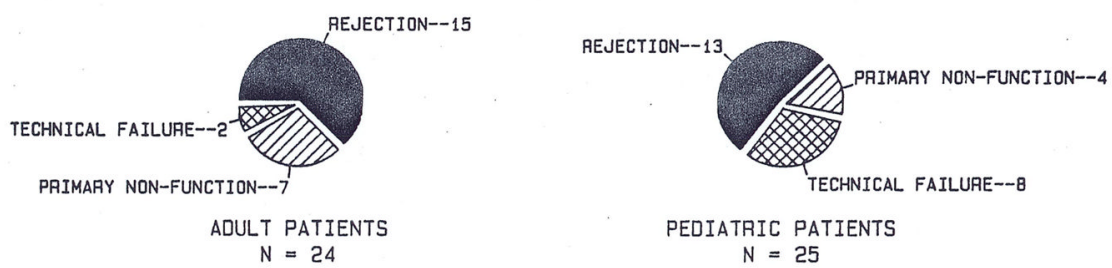

FIG. 4. Indications for retransplantation

(Reproduced with permission from Shaw et al. ${ }^{1}$ ) 


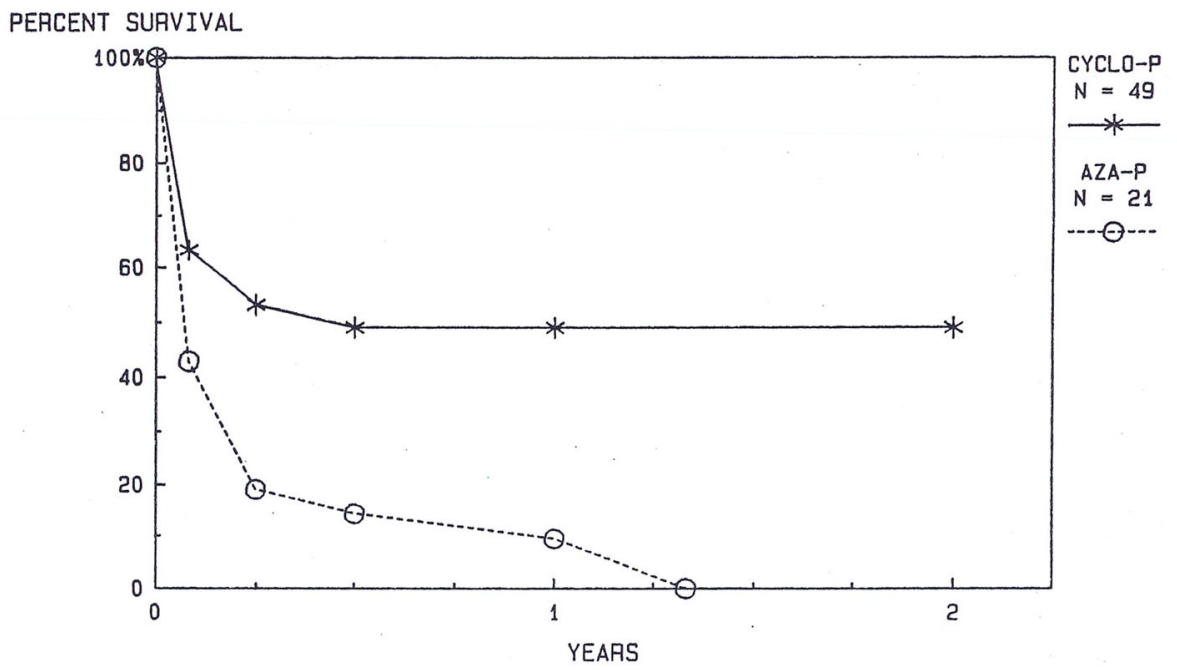

FIG. 5. Actuarial survival after liver retransplantation; before and after the introduction of cyclosporine-prednisone therapy

(Reproduced with permission from Shaw et al. ${ }^{1}$ ) 
PER CENT SUAVIVAL

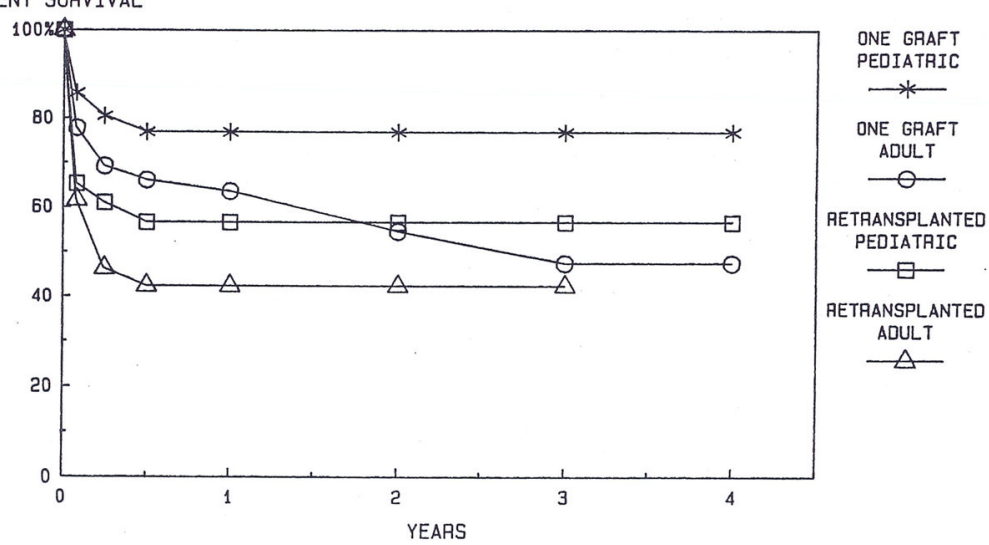

FIG. 6. Actuarial survival after liver retransplantation with cyclosporine and prednisone in pediatric and adult patients

(Reproduced with permission from Shaw et al. ${ }^{1}$ ) 
PEACENT SUAVIVAL

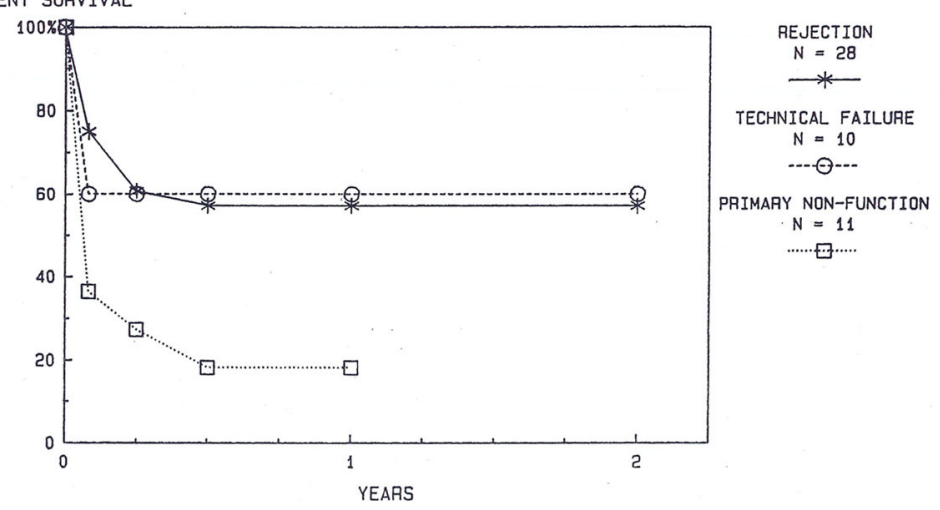

FIG. 7. Actuarial survival for liver transplantation, based on indication for graft replacement (Reproduced with permission from Shaw et al. ${ }^{1}$ ) 


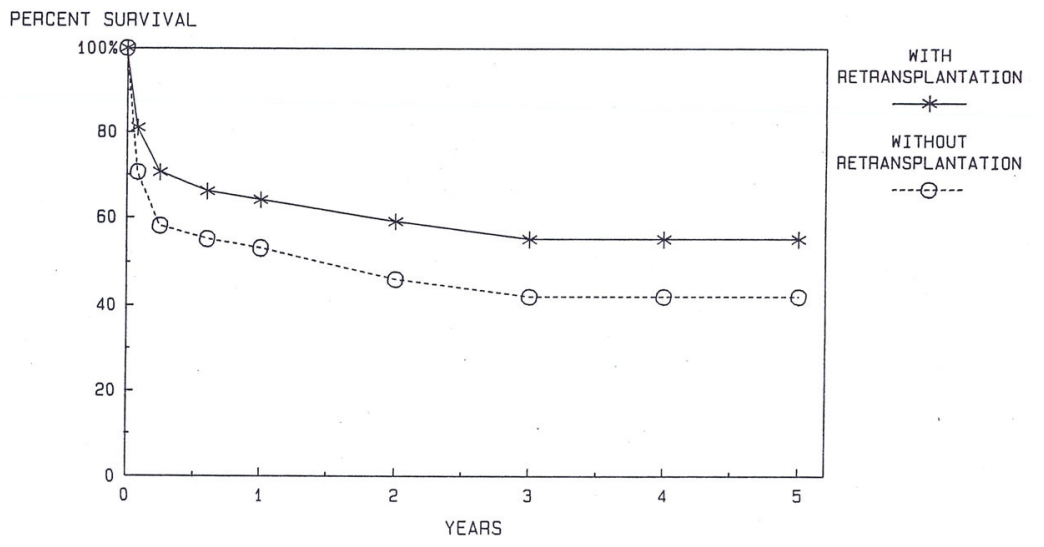

FIG. 8. Effect of liver retransplantation on actuarial survival (Reproduced with permission from Shaw et al. ${ }^{1}$ ) 


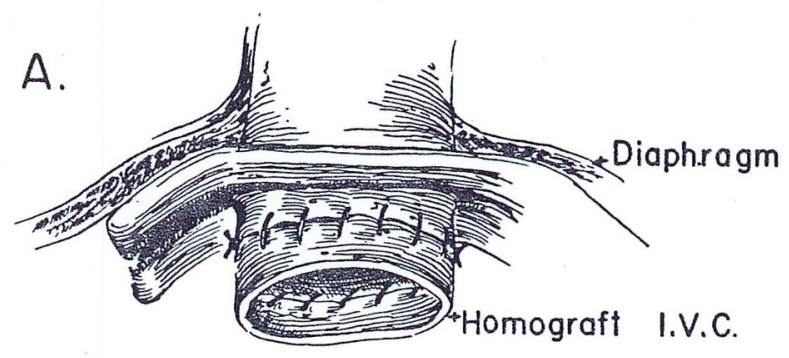

B.

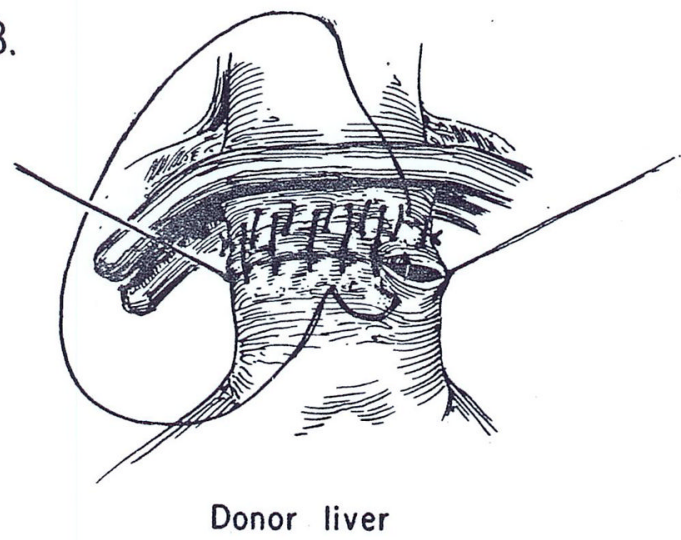

FIG. 9. Intact upper caval anastomosis with short cuff of vein from the liver being removed, which is left in place for anastomosis with the new organ

(Reproduced with permission from Starzl. ${ }^{6}$ ) 


\section{TABLE 1}

Seven Patients for Whom Donors Were Not Available in Time

\begin{tabular}{ll}
\hline Cause of Graft Failure & Cause of Death \\
\hline Technical & Hepatic gangrene \\
Technical & Hemorrhage \\
Primary nonfunction & Hepatic failure \\
Acute rejection & Hepatic failure \\
Acute rejection & Hepatic failure \\
Chronic rejection & Sepsis \\
Chronic rejection & Sepsis \\
\hline
\end{tabular}


TABLE 2

Operative Blood Use: First Versus Second Hepatic Transplantation 1983 to 1984

\begin{tabular}{lccc}
\hline & First Graft & Second Graft & p Value \\
\hline Adults $(\mathrm{n}=20)$ & & \\
Mean & $37.9 \pm 37.2^{*}$ & $16.5 \pm 12.9$ & 0.05 \\
Median & 29 & 16 & 0.02 \\
Range & $4-157$ & $1-56$ & \\
Children $(\mathrm{n}=16)$ & & 0.05 \\
Mean & $10.9 \pm 7.0$ & $5.5 \pm 2.5(9.7 \pm 10.6)^{\dagger}$ & 0.01 \\
Median & 10 & 15 & \\
Range & $3-25(2-36)^{\dagger}$ & $2-11$ & \\
\hline
\end{tabular}

*Values expressed are units of packed red blood cells.

${ }^{\dagger}$ If cases of arterial thrombosis are included (see Discussion). 
TABLE 3

Hepatic Retransplantation: Influence of Donor-Specific Cytotoxic T-Cell Antibodies

\begin{tabular}{lcrr}
\hline & Negative Cross-Match & Positive Cross-Match & Not Done \\
\hline No retransplant & 94 & 21 & 76 \\
All retransplants & 27 & 6 & 16 \\
Rejected retransplants & 16 & 6 & 8 \\
\hline
\end{tabular}


TABLE 4

Tertiary Hepatic Transplantation as of August 1, 1984

\begin{tabular}{lll}
\hline & Dead & Alive \\
\hline Interval from second to third transplant & $7,10,19,57$ days & $13,28,41,120,264$ days \\
Survival after third transplant & $9,10,14,43$ days & $7,9,11,17,18$ months \\
\hline
\end{tabular}

